\title{
EVOLUTION EQUATIONS IN DISCRETE AND CONTINUOUS TIME FOR NONEXPANSIVE OPERATORS IN BANACH SPACES
}

\author{
Guillaume Vigeral ${ }^{1}$
}

\begin{abstract}
We consider some discrete and continuous dynamics in a Banach space involving a non expansive operator $J$ and a corresponding family of strictly contracting operators $\Phi(\lambda, x):=\lambda J\left(\frac{1-\lambda}{\lambda} x\right)$ for $\lambda \in] 0,1]$. Our motivation comes from the study of two-player zero-sum repeated games, where the value of the $n$-stage game (resp. the value of the $\lambda$-discounted game) satisfies the relation $v_{n}=$ $\Phi\left(\frac{1}{n}, v_{n-1}\right)$ (resp. $v_{\lambda}=\Phi\left(\lambda, v_{\lambda}\right)$ ) where $J$ is the Shapley operator of the game. We study the evolution equation $u^{\prime}(t)=J(u(t))-u(t)$ as well as associated Eulerian schemes, establishing a new exponential formula and a Kobayashi-like inequality for such trajectories. We prove that the solution of the nonautonomous evolution equation $u^{\prime}(t)=\Phi(\boldsymbol{\lambda}(t), u(t))-u(t)$ has the same asymptotic behavior (even when it diverges) as the sequence $v_{n}$ (resp. as the family $v_{\lambda}$ ) when $\boldsymbol{\lambda}(t)=1 / t$ (resp. when $\boldsymbol{\lambda}(t)$ converges slowly enough to 0$)$.
\end{abstract}

Mathematics Subject Classification. 47H09, 47J35, 34E10.

Received November 4, 2008. Revised March 18, 2009.

Published online July 31, 2009.

\section{INTRODUCTION}

The topic of the asymptotic behavior of trajectories defined through nonexpansive mappings in Banach spaces arise in numerous domains such as nonlinear semigroups theory [3,6,7,12,13,17,21], game theory [15,19,20,24-26] as well as in discrete events systems [9-11].

Given a nonexpansive function $J$ from a Banach space $X$ to itself, evolution equation

$$
U^{\prime}(t)=J(U(t))-U(t)
$$

is a particular case of the widely-studied

$$
U^{\prime}(t) \in-A(U(t))
$$

for a maximal monotone operator $A$. Typically, the study of the asymptotics for such evolution equation and its Eulerian and proximal discretizations has been made in Hilbert spaces [6] or at least assuming some geometric properties in the case of Banach spaces [15,21]. Another usual assumption is the non emptiness of the set $A^{-1}(0)$.

Keywords and phrases. Banach spaces, nonexpansive mappings, evolution equations, asymptotic behavior, Shapley operator.

1 Équipe Combinatoire et Optimisation, CNRS FRE3232, Université Pierre et Marie Curie, Paris 6, UFR 929, 175 rue du Chevaleret, 75013 Paris, France. guillaumevigeral@gmail.com 
On the other hand, in the framework of two-person zero-sum games repeated in discrete time, the values $v_{n}$ and $v_{\lambda}$ of the $n$-stage (resp. $\lambda$-discounted) game satisfy respectively:

$$
\begin{gathered}
v_{n}=\frac{J^{n}(0)}{n}=\Phi\left(\frac{1}{n}, v_{n-1}\right) \\
v_{\lambda}=\Phi\left(\lambda, v_{\lambda}\right)
\end{gathered}
$$

where $J$ is the so-called Shapley operator of the game and $\Phi(\lambda, x):=\lambda J\left(\frac{1-\lambda}{\lambda} x\right)$. This operator $J$ is nonexpansive for the uniform norm, hence $A=I-J$ is a maximal monotone operator in the sense of [12]. However two unusual facts appears in the study of the asymptotics of those values: first $A^{-1}(0)$, the set of fixed points of $J$, is generally empty. Another difficulty lies in the lack of smoothness of the unit ball $\mathcal{B}_{\|\cdot\|_{\infty}}$, which might induce oscillations of the discrete trajectories defined above [15].

The purpose of this paper is to investigate the relation between several discrete and continuous dynamics in Banach spaces. Because our motivation comes from this game-theoretic framework, we neither make any geometrical assumptions on the unit ball, nor suppose non emptiness of $A^{-1}(0)$. In continuous time, dynamics that we will consider are (1.1) as well as non autonomous evolution equations of the form

$$
u^{\prime}(t)=\Phi(\boldsymbol{\lambda}(t), u(t))-u(t)
$$

for some parameterizations $\boldsymbol{\lambda}$. We establish that the quantities defined in (1.2) and (1.3) behave asymptotically as the solutions of these various evolution equations. Surprisingly this is true not only when there is convergence; even when they oscillate we prove that discrete and continuous trajectories remain asymptotically close.

Section 2 is devoted to definitions and basic results. In Section 3 we study the relation between the solution $U$ of evolution equation (1.1) and related Eulerian schemes, establishing in particular that $\left\|v_{n}-\frac{U(n)}{n}\right\|$ converges to 0 . In the process we prove that some classical results (e.g. exponential formula [7], Kobayashi inequality [13]) involving the proximal trajectories for a maximal monotone operator $A$ have an Eulerian explicit counterpart in the case $A=I-J$. In Section 4 we consider the non autonomous equation (1.4). We show that for $\lambda(t)=\frac{1}{t}$ the solution behave asymptotically as the sequence $v_{n}$, and that when $\boldsymbol{\lambda}$ converges slowly enough to 0 the solution behave asymptotically as the family $v_{\lambda}$.

\section{Discrete time MOdel}

\subsection{Nonexpansive operators}

Let $(X,\|\cdot\|)$ be a Banach space, and $J$ a nonexpansive mapping from $X$ into itself :

$$
\|J(x)-J(y)\| \leq\|x-y\| \quad \forall(x, y) \in X^{2} .
$$

We define, for $n \in \mathbb{N}$ and $\lambda \in] 0,1]$,

$$
\begin{aligned}
V_{n} & =J\left(V_{n-1}\right)=J^{n}(0) \\
V_{\lambda} & =J\left((1-\lambda) V_{\lambda}\right) .
\end{aligned}
$$

Notice that $V_{\lambda}$ is well-defined because $J((1-\lambda) \cdot)$ is strictly contracting, hence has a unique fixed point.

Example 2.1. For any $c \in \mathbb{R}$, the mapping $J$ from $\mathbb{R}$ to itself defined by $J(x)=x+c$ is nonexpansive. In that case, $V_{n}=n c$ and $V_{\lambda}=\frac{c}{\lambda}$.

These quantities being unbounded in general (see above), we also introduce their normalized versions

$$
\begin{aligned}
& v_{n}=\frac{V_{n}}{n} \\
& v_{\lambda}=\lambda V_{\lambda} .
\end{aligned}
$$


In the previous example, one gets $v_{n}=v_{\lambda}=c$ for all $n$ and $\lambda$. In general it is easy to prove that these normalized quantities are bounded:

Lemma 2.2. For any $n \in \mathbb{N}$ and $\lambda \in] 0,1]$,

$$
\begin{aligned}
\left\|v_{n}\right\| & \leq\|J(0)\| \\
\left\|v_{\lambda}\right\| & \leq\|J(0)\| .
\end{aligned}
$$

Proof. Since $J$ is non expansive,

$$
\left\|V_{n}-V_{n-1}\right\|=\left\|J\left(V_{n-1}\right)-J\left(V_{n-2}\right)\right\| \leq\left\|V_{n-1}-V_{n-2}\right\| .
$$

By induction this implies that

$$
\left\|V_{n}\right\| \leq n\left\|V_{1}\right\|=n\|J(0)\|
$$

On the other hand, again using the fact that $J$ is non expansive,

$$
\begin{aligned}
\left\|V_{\lambda}\right\|-\|J(0)\| & \leq\left\|V_{\lambda}-J(0)\right\| \\
& =\left\|J\left((1-\lambda) V_{\lambda}\right)-J(0)\right\| \\
& \leq(1-\lambda)\left\|V_{\lambda}\right\|
\end{aligned}
$$

and so

$$
\left\|v_{\lambda}\right\|=\lambda\left\|V_{\lambda}\right\| \leq\|J(0)\|
$$

To underline the link between the families $\left\{v_{n}\right\}_{n \in \mathbb{N}}$ and $\left\{v_{\lambda}\right\}_{\lambda \in] 0,1]}$ it is also of interest to introduce the family of strictly contracting operators $\Phi(\lambda, \cdot), \lambda \in] 0,1]$, defined by

$$
\Phi(\lambda, x)=\lambda J\left(\frac{1-\lambda}{\lambda} x\right) .
$$

The function $\Phi(\lambda, \cdot)$ can be seen as a perturbed recession function of $J$ : because of the nonexpansiveness of $J$,

$$
\lim _{\lambda \rightarrow 0} \Phi(\lambda, x)=\lim _{\lambda \rightarrow 0} \lambda J\left(\frac{x}{\lambda}\right)=\lim _{t \rightarrow+\infty} \frac{J(t x)}{t}
$$

which is the definition of the recession function of $J[23]$.

The quantities $v_{n}$ and $v_{\lambda}$ then satisfy the relations

$$
\begin{aligned}
& v_{n}=\Phi\left(\frac{1}{n}, v_{n-1}\right) ; v_{0}=0 \\
& v_{\lambda}=\Phi\left(\lambda, v_{\lambda}\right) .
\end{aligned}
$$

Notice that since $\Phi(\lambda, \cdot)$ is strictly contracting, any sequence $w_{n} \in X$ satisfying

$$
w_{n}=\Phi\left(\lambda, w_{n-1}\right)
$$

converges strongly to $v_{\lambda}$ as $n$ goes to $+\infty$. 


\subsection{Shapley operators}

An important application, which is our main motivation, is obtained in the framework of zero-sum two player repeated games [25]. For example take the simple case of a stochastic game with a finite state space $\Omega$, compact move sets $S$ and $T$ for player 1 and 2 respectively, payoff $g$ from $S \times T \times \Omega$ to $\mathbb{R}$, and transition probability $\rho$ from $S \times T \times \Omega$ to $\Delta(\Omega)$ (the set of probabilities on $\Omega$ ). Let $\mathcal{S}=\Delta_{f}(S)$ (resp. $\mathcal{T}=\Delta_{f}(T)$ ) the sets of probabilities on $S$ (resp. $T$ ) with finite support; we still denote by $g$ and $\rho$ the multilinear extensions from $S \times T$ to $\mathcal{S} \times \mathcal{T}$ of the corresponding functions.

The game is played as follows: an initial stage $\omega_{1} \in \Omega$ is given, known by each player. At each stage $m$, knowing past history and current state $\omega_{m}$, player 1 (resp. player 2) chooses $\sigma \in \mathcal{S}$ (resp. $\tau \in \mathcal{T}$ ). A move $a_{m}$ of player 1 (resp. $b_{m}$ of player 2 ) is drawn accordingly to $\sigma$ (resp. $\tau$ ). The payoff $g_{m}$ at stage $m$ is then $g\left(a_{m}, b_{m}, \omega_{m}\right)$ and $\omega_{m+1}$, the state at stage $m+1$, is drawn accordingly to $\rho\left(a_{m}, b_{m}, \omega_{m}\right)$.

There are several ways of evaluating a payoff for a given infinite history:

$-\frac{1}{n} \sum_{m=1}^{n} g_{m}$ is the payoff of the $n$-stage game;

$-\lambda \sum_{m=1}^{+\infty}(1-\lambda)^{i-1} g_{m}$ is the payoff of the $\lambda$-discounted game.

For a given initial state $\omega$, we denote the values of those games by $v_{n}(\omega)$ and $v_{\lambda}(\omega)$ respectively; $v_{n}$ and $v_{\lambda}$ are thus functions from $\Omega$ into $\mathbb{R}$.

Let $\mathcal{F}=\{f: \Omega \longrightarrow \mathbb{R}\}$; the Shapley operator $J$ from $\mathcal{F}$ to itself is then defined by $f \rightarrow J(f)$, where $J(f)$ is the function from $\Omega$ to $\mathbb{R}$ satisfying

$$
\begin{aligned}
J(f)(\omega) & =\sup _{\sigma \in \mathcal{S}} \inf _{\tau \in \mathcal{T}}\left\{g(\sigma, \tau, \omega)+\sum_{\omega^{\prime} \in \Omega} f\left(w^{\prime}\right) \rho\left(\omega^{\prime} \mid \sigma, \tau, \omega\right)\right\} \\
& =\inf _{\tau \in \mathcal{T}} \sup _{\sigma \in \mathcal{S}}\left\{g(\sigma, \tau, \omega)+\sum_{\omega^{\prime} \in \Omega} f\left(w^{\prime}\right) \rho\left(\omega^{\prime} \mid \sigma, \tau, \omega\right)\right\} .
\end{aligned}
$$

Then $J$ is nonexpansive on $\mathcal{F}$ endowed with the uniform norm. The value $v_{n}$ of the $n$-stage game (resp. the value $v_{\lambda}$ of the $\lambda$-discounted game) satisfies relation (2.9) (resp. (2.10)).

This recursive structure holds in a wide class of zero-sum repeated games and the study of the asymptotic behavior of $v_{n}$ (resp. $v_{\lambda}$ ) as $n$ tends to $+\infty$ (resp. as $\lambda$ tends to 0 ) is a major topic in game theory (see [25] for example). Convergence of both $v_{n}$ and $v_{\lambda}$ (as well as equality of the limits) has been obtained for different class of games, for example absorbing games [14], recursive games [8], games with incomplete information [2], finite stochastic games [4,5], and Markov Chain Games with incomplete information [22].

Even in the simple case of a finite stochastic game where the space $\mathcal{F}$ on which $J$ is defined is $\mathbb{R}^{n}$, the Shapley operator $J$ is only nonexpansive for the uniform norm $\ell^{\infty}$. In the case of a general Shapley operator $J$, the Banach space (which may be infinite dimensional) on which $J$ is nonexpansive is always a set of bounded real functions (defined on a set $\Omega$ of states) endowed with the uniform norm. As shown in $[11,15]$, this lack of geometrical smoothness implies that the families $v_{n}$ and $v_{\lambda}$ may not converge. They may also converge to two different limits [16]. However the goal of the so called "Operator Approach" (see [24,26]) is to infer, from specific properties in the framework of games, convergence of both $v_{n}$ and $v_{\lambda}$ as well as equality of their limits.

A closely related application, in the framework of discrete event systems, is the problem of existence of the cycle-time of a topical mapping $[9,10]$.

\subsection{Associated evolution equations}

In the current paper we investigate a slightly different direction: the aim is to show that the sequence $v_{n}$ and the family $v_{\lambda}$ defined in equations (2.9) and (2.10) behave asymptotically as the solutions of certain continuoustime evolution equations. This is interesting for at least three reasons: first, this implies that proving the convergence of $v_{n}$ or $v_{\lambda}$ reduces to study the asymptotic of the solution of some evolution equation. Second, even if the definitions (2.9) of $v_{n}$ and (2.10) of $v_{\lambda}$ may seem dissimilar since one is recursive and the other is 
a fixed point equation, we will see that the corresponding equations in continuous time are of the same kind, hence it gives an insight on the equality $\lim v_{n}=\lim v_{\lambda}$, satisfied for a wide class of games. Third, we will prove in the process some results of interest in their own right.

Notice that equation (2.1) can also be written as a difference equation

$$
\left(V_{n+1}-V_{n}\right)=J\left(V_{n}\right)-V_{n}
$$

which can be viewed as a discrete version of the evolution equation

$$
U^{\prime}(t)=J(U(t))-U(t)
$$

Similarly, equations (2.9) and (2.11) can be considered as discrete versions of

$$
u^{\prime}(t)=\Phi\left(\frac{1}{t+1}, u(t)\right)-u(t)
$$

and

$$
u^{\prime}(t)=\Phi(\lambda, u(t))-u(t)
$$

respectively. Notice that while (2.17) is autonomous, (2.16) is not.

The asymptotic relation between solutions of (2.15) and (2.1) will be discussed in Section 3. In that section we will a also prove some results about Eulerian schemes related to (2.15), which have an interpretation in terms of games with uncertain duration $[19,20]$ in the case of a Shapley Operator.

In Section 4, we will study the asymptotic behavior of solutions of the non-autonomous evolution equation

$$
u^{\prime}(t)=\Phi(\boldsymbol{\lambda}(t), u(t))-u(t)
$$

for some time-dependent parameterizations $\boldsymbol{\lambda}(t)$, which in particular will cover both cases of equations (2.16) and (2.17). We will first prove that when $\boldsymbol{\lambda}(t)=\frac{1}{t}$ the solution of (2.18) has the same asymptotic behavior, as $t$ goes to $+\infty$, as the sequence $v_{n}$ as $n$ goes to $+\infty$. We will then examine the case where the parameterization $\lambda(t)$ converges slowly enough to 0 , establishing that the solution of (2.18) has then the same asymptotic behavior as the family $v_{\lambda}$ as $\lambda$ goes to 0 . Finally, using our results in continuous time, we will study other dynamics in discrete time generalizing (2.9) and (2.11). Similarly to Section 3, in the case of a Shapley operator these dynamics have an interpretation in terms of games with uncertain duration.

\section{Dynamical System Related to the operator $J$}

Let us denote $A=I-J$; the operator $A$ is $m$-accretive, meaning that for any $\lambda>0$ both properties are satisfied:

(i) $\|x-y+\lambda A(x)-\lambda A(y)\| \geq\|x-y\|$ for all $(x, y) \in X^{2}$.

(ii) $I+\lambda A$ is surjective.

This implies that $A$ is maximal monotone [12]. Recall that the analogous in continuous time of equation (2.1) defining $V_{n}$ is evolution equation (2.15), which can also be written as

$$
U^{\prime}(t)=-A(U(t))
$$

with initial condition $U(0)=U_{0}$, the Cauchy-Lipschitz theorem ensuring the existence and uniqueness of such a solution.

Example 3.1. Following Example 2.1, suppose $J(x)=x+c$. Then one has $A(x)=-c$, so $U(t)=U_{0}+c t$. 
This simple example shows that, as in discrete time where the true sequence to consider is not $V_{n}$ but the normalized $v_{n}$, we are not expecting convergence of $U(t)$ but rather of the normalized quantity $\frac{U(t)}{t}$. This is a consequence of the fact that we do not assume non emptiness of $A^{-1}(0)$.

Apart from equation (2.1), there are numerous other natural discretizations of equation (3.1). For every $x_{0} \in X$ and any sequence $\left\{\lambda_{n}\right\}$ in $[0,1]^{2}$ the explicit Eulerian scheme is defined by

$$
x_{n}-x_{n-1}=-\lambda_{n} A x_{n-1}
$$

that is

$$
x_{n}=\left(\prod_{i=n}^{1}\left[I-\lambda_{i} A\right]\right)\left(x_{0}\right) .
$$

Notice that choosing $x_{0}=0$ and $\lambda_{n}=1$ for all $n$ leads to the definition (2.1) of $V_{n}$.

Other discrete trajectories are implicit proximal schemes (first introduced when $A=\partial f$ in [18]) which satisfy:

$$
x_{n}-x_{n-1}=-\lambda_{n} A x_{n}
$$

that is

In both cases we denote

$$
x_{n}=\left(\prod_{i=n}^{1}\left[I+\lambda_{i} A\right]^{-1}\right)\left(x_{0}\right) .
$$

$$
\begin{aligned}
\sigma_{n} & =\sum_{i=1}^{n} \lambda_{i} \\
\tau_{n} & =\sum_{i=1}^{n} \lambda_{i}^{2}
\end{aligned}
$$

Usually proximal schemes share better asymptotic properties (take the simple example where $A$ is a rotation in $\mathbb{R}^{2}$ and $\lambda_{n} \notin \ell^{2}$ : then the proximal scheme will converge to the fixed point of the rotation, while the Eulerian one will diverge). However Eulerian schemes have the remarkable feature that they can be computed explicitly, and they arise naturally in the game-theoretic framework:

Example 3.2. When $J$ is the Shapley operator of a stochastic game $\Gamma, x_{n}$ defined by (3.2) is the non-normalized value of the following $n$-stage game: states, actions, payoff and transition are as in $\Gamma$, but at stage 1 there is a probability $1-\lambda_{n}$ that the game goes on to stage 2 without any payoff or transition. Similarly at stage 2 , there is no payoff nor transition with probability $1-\lambda_{n-1}$, and at stage $n$ with probability $1-\lambda_{1}$. In that case $\sigma_{n}$ and $\tau_{n}$ have a nice interpretation: the expected number of stages really played is $\sigma_{n}$, and the variance is $\sigma_{n}-\tau_{n}$. It is also worthwhile to notice that such games are particular cases of stochastic games with uncertain duration $[19,20]$.

For this reason we will study exclusively Eulerian schemes, in the case of an operator $A=I-J$. Results of this section will be of three kind: first we study the relative behavior of continuous and discrete dynamics when time goes to infinity. Given a sequence $\lambda_{n} \notin \ell^{1}$ one investigates the asymptotic relation between $U\left(\sigma_{n}\right)$ and the $n$-th term $x_{n}$ of the Eulerian scheme defined in (3.2). This is done first in the special case of $V_{n}$ (Cor. 3.8) and then in general (Cor. 3.13).

We also consider the case of a fixed time $t$. In that case one cuts the interval $[0, t]$ in a finite number $m$ of intervals of length $\lambda_{i}$. These steps define an explicit scheme by (3.2), hence an approximate trajectory by linear interpolation. One expects such a trajectory to be asymptotically closer to the continuous trajectory defined

\footnotetext{
${ }^{2}$ Usually these schemes are defined for any sequence of positive steps, but here, since we need the operators $I-\lambda_{n} A$ to be non expansive, we have to assume that the $\lambda_{n}$ lie in $[0,1]$.
} 
by (3.1) as the discretization of the interval becomes finer. This is proved first in the case where $\lambda_{i}=\frac{t}{m}$ for $1 \leq i \leq m$ (Prop. 3.10), and then generalized in Proposition 3.14.

In the process we prove that two classical results, involving proximal schemes and holding for any maximal monotone operator, have an Eulerian counterpart when $A$ is of the form $I-J$ : we establish a new exponential formula in Proposition 3.10 and a Kobayashi-like inequality in Proposition 3.11.

\subsection{Asymptotic study of the trajectory defined by equation (3.1)}

The study of the asymptotic behavior of the solution of equation (2.15) in general Banach spaces has started in the early 70's, in particular the main result of this subsection, Corollary 3.8 relating $v_{n}$ and $\frac{U(n)}{n}$, is already known (see [3,17]). Here we prove it in a different way, similar to the first chapter of [6], establishing during the proof some inequalities that will be helpful in the remaining of the paper.

Let us begin by proving several useful lemmas:

Lemma 3.3. Let $f$ be a continuous function from $[a, b] \subset \mathbb{R}$ to $\mathbb{R}$ such that for every $t \in[a, b]$

$$
f(t) \leq M+\int_{a}^{t}[g(s)+\beta(s) f(s)] \mathrm{d} s
$$

for some continuous function $g$ and some non-negative measurable function $\beta$ such that $\int_{a}^{b} \beta(s) \mathrm{d} s<+\infty$.

Then $f$ satisfies

$$
f(t) \leq \mathrm{e}^{\int_{a}^{t} \beta(s) \mathrm{d} s}\left(M+\int_{a}^{t} g(s) \mathrm{e}^{-\int_{a}^{s} \beta(r) \mathrm{d} r} \mathrm{~d} s\right)
$$

for all $t \in[a, b]$.

Proof. Define $\alpha(t)=M+\int_{a}^{t} g(s) \mathrm{d} s$. Since $f(t) \leq \alpha(t)+\int_{a}^{t} \beta(s) f(s) \mathrm{d} s$, Gronwall's inequality ([27], p. 15) implies that

$$
f(t) \leq \alpha(t)+\mathrm{e}^{\int_{a}^{t} \beta(s) \mathrm{d} s} \cdot \int_{a}^{t} \alpha(s) \beta(s) \mathrm{e}^{-\int_{a}^{s} \beta(r) \mathrm{d} r} \mathrm{~d} s .
$$

Integrating by part the last integral gives

$$
\begin{aligned}
f(t) & \leq \alpha(t)+\mathrm{e}^{\int_{a}^{t} \beta(s) \mathrm{d} s} \cdot\left(\alpha(0)-\alpha(t) \mathrm{e}^{-\int_{a}^{t} \beta(s) \mathrm{d} s}+\int_{a}^{t} \alpha^{\prime}(s) \mathrm{e}^{-\int_{a}^{s} \beta(r) \mathrm{d} r} \mathrm{~d} s\right) \\
& =\mathrm{e}^{\int_{a}^{t} \beta(s) \mathrm{d} s}\left(M+\int_{a}^{t} g(s) \mathrm{e}^{-\int_{a}^{s} \beta(r) \mathrm{d} r} \mathrm{~d} s\right) .
\end{aligned}
$$

In the remaining of the paper we will repeatedly use the following consequence of Lemma 3.3:

Proposition 3.4. If $y:[a, b] \subset \mathbb{R} \rightarrow X$ is an absolutely continuous function satisfying for every $t \in[a, b]$

$$
\left\|y(t)+y^{\prime}(t)\right\| \leq(1-\gamma(t))\|y(t)\|+h(t)
$$

where $\gamma$ is a continuous function from $[a, b]$ to $[-\infty, 1]$ and $h$ is a continuous function from $[a, b]$ to $\mathbb{R}$, then $y$ satisfies

$$
\|y(t)\| \leq \mathrm{e}^{-\int_{a}^{t} \gamma(s) \mathrm{d} s}\left(\|y(a)\|+\int_{a}^{t} h(s) \mathrm{e}^{\int_{a}^{s} \gamma(r) \mathrm{d} r} \mathrm{~d} s\right)
$$

for all $t \in[a, b]$. 
Proof. $z(t)=y(t) \mathrm{e}^{t}$ satisfies $\left\|z^{\prime}(t)\right\| \leq(1-\gamma(t))\|z(t)\|+h(t) \mathrm{e}^{t}$, hence for every $t \in[a, b]$

$$
\begin{aligned}
\|z(t)\| & \leq\|z(a)\|+\left\|\int_{a}^{t} z^{\prime}(s) \mathrm{d} s\right\| \\
& \leq\|z(a)\|+\int_{a}^{t}\left\|z^{\prime}(s)\right\| \mathrm{d} s \\
& \leq\|z(a)\|+\int_{a}^{t} h(s) \mathrm{e}^{s}+(1-\gamma(s))\|z(s)\| .
\end{aligned}
$$

Applying Lemma 3.3 to $\|z\|$ thus gives

$$
\|z(t)\| \leq \mathrm{e}^{\int_{a}^{t} 1-\gamma(s) \mathrm{d} s}\left(\|z(a)\|+\int_{a}^{t} h(s) \mathrm{e}^{s} \mathrm{e}^{-\int_{a}^{s} 1-\gamma(r) \mathrm{d} r} \mathrm{~d} s\right) .
$$

Multiplying each side by $\mathrm{e}^{-t}$ implies the result.

We now use this technical result to compare two solutions of (2.15):

Proposition 3.5. If both $U$ and $V$ satisfy (2.15), then $\|U(t)-V(t)\|$ is non-increasing.

Proof. Define $f=U-V$ which satisfies

$$
\left\|f(t)+f^{\prime}(t)\right\|=\|J(U(t))-J(V(t))\| \leq\|U(t)-V(t)\|=\|f(t)\| .
$$

Apply the preceding proposition to $\gamma \equiv 0$ and $f$.

Corollary 3.6. If $U$ is a solution of (2.15), then $\left\|U^{\prime}(t)\right\|$ is non-increasing.

Proof. Let $h>0$ and $U_{h}(t)=U(t+h)$. The function $U_{h}$ satisfies equation (2.15), so applying the preceding proposition to $U$ and $U_{h}$ we get that $t \rightarrow \frac{\|U(t+h)-U(t)\|}{h}$ is non-increasing on $\mathbb{R}^{+}$. Letting $h$ go to 0 gives the result.

An interesting consequence of Corollary 3.6 is the following inequality, proved in Chapter 1 of [6]:

Lemma 3.7 (Chernoff's estimate). Let $U$ be the solution of $(2.15)$ with $U(0)=U_{0}$. Then

$$
\left\|U(t)-J^{n}\left(U_{0}\right)\right\| \leq\left\|U^{\prime}(0)\right\| \sqrt{t+[n-t]^{2}} .
$$

Sketch of proof. Proceed by induction on $n$; the proof for the case $n=0$ comes from the fact that $\left\|U^{\prime}\right\|$ is non-increasing by Corollary 3.6.

In particular if we take $U_{0}=0$ and $t=n$ in Lemma 3.7, we finally get the following corollary relating continuous and discrete trajectories:

Corollary 3.8. The solution $U$ of (2.15) with $U(0)=0$ satisfies

$$
\left\|\frac{U(n)}{n}-v_{n}\right\| \leq \frac{\|J(0)\|}{\sqrt{n}} .
$$

In particular $v_{n}$ converges iff $\frac{U(t)}{t}$ converges, and then the limits are the same. 
Proof. The only point that remains to be shown is that if the sequence $\frac{U(n)}{n}$ converges as $n$ tends to $+\infty$, then so does $\frac{U(t)}{t}$ as $t$ tends to $+\infty$.

Using Corollary 3.6, we obtain

$$
\|U(t)-U([t])\| \leq(t-[t])\left\|U^{\prime}(0)\right\| \leq\left\|U^{\prime}(0)\right\|
$$

which implies that $\frac{U(t)}{t}-\frac{U([t])}{[t]}$ goes to 0 as $t$ tends to $+\infty$.

\subsection{An exponential formula}

When $A$ is a $m$-accretive operator on a Banach space, a fundamental result (see [7], p. 267) is that the solution $U$ of (2.15) satisfies the following exponential formula for every $t \geq 0$, where the convergence is strong:

$$
\lim _{m \rightarrow+\infty}\left(I+\frac{t}{m} A\right)^{-m}\left(U_{0}\right)=U(t)
$$

In the special case where $J$ is a nonexpansive operator and $A=I-J$, we now establish an Eulerian analogous of this classical "proximal exponential formula".

Definition 3.9. For $x \in X, l \in \mathbb{N}$ and $t \in \mathbb{R}^{+}$, let us denote

$$
U_{t}^{m}(x)=\left(I-\frac{t}{m} A\right)^{m}(x)
$$

the $m$ th term of an Eulerian scheme with steps $\frac{t}{m}$.

Proposition 3.10. Let $U_{0} \in X$ and $U$ the solution of (2.15) with $U(0)=U_{0}$. Then if $m \geq t$,

$$
\left\|U_{t}^{m}\left(U_{0}\right)-U(t)\right\| \leq\left\|A\left(U_{0}\right)\right\| \frac{t}{\sqrt{m}} .
$$

In particular, for any $t \geq 0$, the following strong convergence holds:

$$
\lim _{m \rightarrow+\infty}\left(I-\frac{t}{m} A\right)^{m}\left(U_{0}\right)=U(t) .
$$

Proof. For any $\lambda \in[0,1], J_{\lambda}:=\lambda J+(1-\lambda) I=I-\lambda A$ is nonexpansive. Denote by $U_{\lambda}$ the solution of

$$
U_{\lambda}(t)+U_{\lambda}^{\prime}(t)=J_{\lambda}\left(U_{\lambda}(t)\right)
$$

with $U_{\lambda}(0)=U_{0}$. Applying Lemma 3.7 to $U_{\lambda}$ and the nonexpansive operator $J_{\lambda}$ :

$$
\left\|U_{\lambda}(t)-J_{\lambda}^{n}\left(U_{0}\right)\right\| \leq\left\|U_{\lambda}^{\prime}(0)\right\| \sqrt{t+[n-t]^{2}}
$$

so in particular for $n=t$

$$
\left\|U_{\lambda}(n)-J_{\lambda}^{n}\left(U_{0}\right)\right\| \leq\left\|U_{\lambda}^{\prime}(0)\right\| \sqrt{n} .
$$

Denote by $U$ the the solution of (2.15) with $U(0)=U_{0}$ and notice that the function $t \rightarrow U(\lambda t)$ satisfies (3.10) and has the same initial condition as $U_{\lambda}$. This implies that $U_{\lambda}(t)=U(\lambda t)$ and putting this in (3.11),

$$
\left\|U(\lambda n)-J_{\lambda}^{n}\left(U_{0}\right)\right\| \leq \lambda\left\|U^{\prime}(0)\right\| \sqrt{n} .
$$


For any $t^{\prime} \leq n$, choosing $\lambda=\frac{t^{\prime}}{n} \in[0,1]$ thus gives

$$
\left\|U\left(t^{\prime}\right)-\left(I-\frac{t^{\prime}}{n} A\right)^{n}\left(U_{0}\right)\right\| \leq\left\|U^{\prime}(0)\right\| \frac{t^{\prime}}{\sqrt{n}}
$$

which is the desired result.

\subsection{Comparison of two Eulerian schemes}

To generalize Proposition 3.10 to explicit schemes with arbitrary steps, it is useful to estimate first the difference between two Euler schemes: let $x_{0}$ and $\hat{x}_{0}$ in $X,\{\lambda\}_{n}$ and $\left\{\hat{\lambda}_{n}\right\}$ two sequences in $\left.] 0,1\right]$. Define $x_{n}, \sigma_{n}$ and $\tau_{n}$ (resp. $\hat{x}_{n}, \hat{\sigma}_{n}$ and $\hat{\tau_{n}}$ ) as in (3.2), (3.4) and (3.5). The following proposition, which gives a majoration of the distance between two Eulerian trajectories, is an analogous of the classical Kobayashi inequality (Lem. 2.1 in [13]) which gives a majoration of the distance between two proximal trajectories:

Proposition 3.11. For any $z \in X$ and $(k, l) \in \mathbb{N}^{2}$,

$$
\left\|x_{k}-\hat{x}_{l}\right\| \leq\left\|x_{0}-z\right\|+\left\|\hat{x}_{0}-z\right\|+\|A(z)\| \sqrt{\left(\sigma_{k}-\hat{\sigma}_{l}\right)^{2}+\tau_{k}+\hat{\tau}_{l}} .
$$

Proof. We proceed by induction and begin by the case $l=0$.

We recall that $J_{\lambda}=I_{\lambda} A$ is non-expansive for $\lambda \leq 1$, so we obtain

$$
\begin{aligned}
\left\|x_{j}-z\right\| & \leq\left\|x_{j}-J_{\lambda_{j}}(z)\right\|+\left\|J_{\lambda_{j}}(z)-z\right\| \\
& =\left\|J_{\lambda_{j}}\left(x_{j-1}\right)-J_{\lambda_{j}}(z)\right\|+\lambda_{j}\|A(z)\| \\
& \leq\left\|x_{j-1}-z\right\|+\lambda_{j}\|A(z)\|
\end{aligned}
$$

and summing these inequalities for $i \in\{1, \ldots, k\}$ we get $\left\|x_{k}-z\right\| \leq\left\|x_{0}-z\right\|+\sigma_{k}\|A(z)\|$, which implies that

$$
\left\|x_{k}-\hat{x}_{0}\right\| \leq\left\|x_{k}-z\right\|+\left\|\hat{x}_{0}-z\right\| \leq\left\|x_{0}-z\right\|+\left\|\hat{x}_{0}-z\right\|+\sigma_{k}\|A(z)\|
$$

and the proposition holds when $l=0$. The case $k=0$ is proved in the same way.

We will now assume the formula to be true for $(k-1, l),(k, l-1)$ et $(k-1, l-1)$ and deduce that it also holds for $(k, l)$.

Define numbers $\alpha_{k, l}=\frac{\lambda_{k}\left(1-\hat{\lambda}_{l}\right)}{\lambda_{k}+\hat{\lambda}_{l}-\lambda_{k} \hat{\lambda}_{l}}, \beta_{k, l}=\frac{\hat{\lambda}_{l}\left(1-\lambda_{k}\right)}{\lambda_{k}+\hat{\lambda}_{l}-\lambda_{k} \hat{\lambda}_{l}}$ et $\gamma_{k, l}=\frac{\lambda_{k} \hat{\lambda}_{l}}{\lambda_{k}+\hat{\lambda}_{l}-\lambda_{k} \hat{\lambda}_{l}}$ and note that they are nonnegative with sum 1 . Introduce also $c_{k, l}=\sqrt{\left(\sigma_{k}-\hat{\sigma}_{l}\right)^{2}+\tau_{k}+\hat{\tau}_{l}}$. For any $x$ and $y$ in $X$, one check that the following equality holds:

$$
J_{\lambda_{k}}(x)-J_{\hat{\lambda}_{l}}(y)=\alpha_{k, l}\left(J_{\lambda_{k}}(x)-y\right)+\beta_{k, l}\left(x-J_{\hat{\lambda}_{l}}(y)\right)+\gamma_{k, l}(J(x)-J(y)) .
$$

In particular, letting $x=x_{k-1}, y=\hat{x}_{l-1}$ and using the non-expansiveness of $J$, we get

$$
\left\|x_{k}-\hat{x}_{l}\right\| \leq \alpha_{k, l}\left\|x_{k}-\hat{x}_{l-1}\right\|+\beta_{k, l}\left\|x_{k-1}-\hat{x}_{l}\right\|+\gamma_{k, l}\left\|x_{k-1}-\hat{x}_{l-1}\right\|
$$

so by induction,

$$
\begin{aligned}
\left\|x_{k}-\hat{x}_{l}\right\| & \leq\left\|x_{0}-z\right\|+\left\|\hat{x}_{0}-z\right\|+\|A(z)\|\left(\alpha_{k, l} c_{k, l-1}+\beta_{k, l} c_{k-1, l}+\gamma_{k, l} c_{k-1, l-1}\right) \\
& \leq\left\|x_{0}-z\right\|+\left\|\hat{x}_{0}-z\right\|+\|A(z)\| \sqrt{\alpha_{k, l}+\beta_{k, l}+\gamma_{k, l}} \sqrt{d_{k, l}} \\
& =\left\|x_{0}-z\right\|+\left\|\hat{x}_{0}-z\right\|+\|A(z)\| \sqrt{d_{k, l}}
\end{aligned}
$$

where we have denoted $d_{k, l}=\alpha_{k, l} c_{k, l-1}^{2}+\beta_{k, l} c_{k-1, l}^{2}+\gamma_{k, l} c_{k-1, l-1}^{2}$. 
In addition,

$$
\begin{aligned}
c_{k, l-1}^{2} & =\left(\sigma_{k}-\hat{\sigma}_{l-1}\right)^{2}+\tau_{k}+\hat{\tau}_{l-1} \\
& =\left(\sigma_{k}-\hat{\sigma}_{l}+\hat{\lambda}_{l}\right)^{2}+\tau_{k}+\hat{\tau}_{l-1} \\
& =\left(\sigma_{k}-\hat{\sigma}_{l}\right)^{2}+\hat{\lambda}_{l}^{2}+2 \hat{\lambda}_{l}\left(\sigma_{k}-\hat{\sigma}_{l}\right)+\tau_{k}+\hat{\tau}_{l-1} \\
& =c_{k, l}^{2}+2 \hat{\lambda}_{l}\left(\sigma_{k}-\hat{\sigma}_{l}\right)
\end{aligned}
$$

and similarly,

$$
c_{k-1, l}^{2}=c_{k, l}^{2}-2 \lambda_{k}\left(\sigma_{k}-\hat{\sigma}_{l}\right)
$$

Moreover

$$
\begin{aligned}
c_{k-1, l-1}^{2} & =\left(\sigma_{k-1}-\hat{\sigma}_{l-1}\right)^{2}+\tau_{k-1}+\hat{\tau}_{l-1} \\
& =\left(\sigma_{k}-\hat{\sigma}_{l}+\hat{\lambda}_{l}-\lambda_{k}\right)^{2}+\tau_{k-1}+\hat{\tau}_{l-1} \\
& =\left(\sigma_{k}-\hat{\sigma}_{l}\right)^{2}+\hat{\lambda}_{l}^{2}+\lambda_{k}^{2}+2\left(\hat{\lambda}_{l}-\lambda_{k}\right)\left(\sigma_{k}-\hat{\sigma}_{l}\right)-2 \hat{\lambda}_{l} \lambda_{k}+\tau_{k-1}+\hat{\tau}_{l-1} \\
& =c_{k, l}^{2}+2\left(\hat{\lambda}_{l}-\lambda_{k}\right)\left(\sigma_{k}-\hat{\sigma}_{l}\right)-2 \hat{\lambda}_{l} \lambda_{k} .
\end{aligned}
$$

So

$$
\begin{aligned}
d_{k, l} & =\alpha_{k, l} c_{k, l-1}^{2}+\beta_{k, l} c_{k-1, l}^{2}+\gamma_{k, l} c_{k-1, l-1}^{2} \\
& =c_{k, l}^{2}+2\left(\sigma_{k}-\hat{\sigma}_{l}\right)\left(\alpha_{k, l} \hat{\lambda}_{l}-\beta_{k, l} \lambda_{k}+\gamma_{k, l}\left(\hat{\lambda}_{l}-\lambda_{k}\right)\right)-2 \hat{\lambda}_{l} \lambda_{k} \gamma_{k, l} \\
& =c_{k, l}^{2}+2 \frac{\sigma_{k}-\hat{\sigma}_{l}}{\lambda_{k}+\hat{\lambda}_{l}-\lambda_{k} \hat{\lambda}_{l}}\left(\lambda_{k} \hat{\lambda}_{l}\left(1-\hat{\lambda}_{l}\right)-\lambda_{k} \hat{\lambda}_{l}\left(1-\lambda_{k}\right)+\lambda_{k} \hat{\lambda}_{l}\left(\hat{\lambda}_{l}-\lambda_{k}\right)\right)-2 \hat{\lambda}_{l} \lambda_{k} \gamma_{k, l} \\
& =c_{k, l}^{2}-2 \hat{\lambda}_{l} \lambda_{k} \gamma_{k, l} \\
& \leq c_{k, l}^{2}
\end{aligned}
$$

and we have established that

$$
\left\|x_{k}-\hat{x}_{l}\right\| \leq\left\|x_{0}-z\right\|+\left\|\hat{x}_{0}-z\right\|+\|A(z)\| \sqrt{\left(\sigma_{k}-\hat{\sigma}_{l}\right)^{2}+\tau_{k}+\hat{\tau}_{l}}
$$

\subsection{Comparison of an Eulerian scheme to a continuous trajectory}

We now combine the results of the two preceding subsections: Proposition 3.10 comparing the continuous trajectory with a particular Eulerian scheme, and Proposition 3.11 relating any two Eulerian schemes.

Corollary 3.12. Let $\left\{x_{n}\right\}_{n \in \mathbb{N}}$ be an Eulerian scheme as defined in (3.2). Then for any $t \geq 0$ and $k \in \mathbb{N}$,

$$
\left\|x_{k}-U(t)\right\| \leq\left\|x_{0}-U_{0}\right\|+\left\|A\left(U_{0}\right)\right\| \sqrt{\left(\sigma_{k}-t\right)^{2}+\tau_{k}}
$$

Proof. Apply Proposition 3.11 to $x_{k}$ and $U_{t}^{m}\left(U_{0}\right)$ to get

$$
\left\|x_{k}-U_{t}^{m}\left(U_{0}\right)\right\| \leq\left\|x_{0}-U_{0}\right\|+\left\|A\left(U_{0}\right)\right\| \sqrt{\left(\sigma_{k}-t\right)^{2}+\tau_{k}+\frac{t^{2}}{m}}
$$

Let $m$ go to $+\infty$ and use Proposition 3.10. 
This corollary has some interesting consequences in two directions, as it generalizes both Corollary 3.8 and Proposition 3.10. First, it shows that any normalized discrete trajectory behave as the normalized continuous one as time goes to infinity:

Corollary 3.13. For any $t \geq 0$ and any Eulerian scheme $\left\{x_{i}\right\}$ such that $\sigma_{k}=t$,

$$
\frac{\left\|x_{k}-U(t)\right\|}{t} \leq \frac{\left\|x_{0}-U_{0}\right\|+\left\|A\left(U_{0}\right)\right\| \sqrt{t}}{t}
$$

Proof. Apply Corollary 3.12 and use the fact that $\tau_{k} \leq \sigma_{k}$ since all $\lambda_{i}$ are in $[0,1]$.

On the other hand, take now the case of a fixed time $t$. Let $U$ be the solution of (3.1) with initial condition $U(0)=U_{0}$, and let $\left\{x_{i}\right\}_{0 \leq i \leq n}$ defined by (3.2) be an Eulerian scheme with same initial condition $x_{0}=U_{0}$ and $\sigma_{n}=t$. One constructs an approximation $\widetilde{x}$ of the continuous trajectory $U$ on the interval $[0, t]$ by $\widetilde{x}\left(\sigma_{k}\right)=x_{k}$ for $0 \leq k \leq n$, and linear interpolation on intervals $\left[\sigma_{k}, \sigma_{k+1}\right]$. The following proposition states that such approximation $\widetilde{x}$ will becomes asymptotically close to $U$ as the discretization $0 \leq \lambda_{1} \leq \lambda_{1}+\lambda_{2} \leq \ldots \leq \sigma_{n-1} \leq$ $\sigma_{n}=t$ of the interval $[0, t]$ gets finer:

Proposition 3.14. For any $t^{\prime}$ in the interval $[0, t]$,

$$
\left\|\widetilde{x}\left(t^{\prime}\right)-U\left(t^{\prime}\right)\right\| \leq\left\|A\left(U_{0}\right)\right\|(1+(1+\sqrt{2}) t) \cdot \sqrt{\max _{1 \leq i \leq n}\left\{\lambda_{i}\right\}}
$$

Proof. Let $t^{\prime} \in[0, t]$ and $k$ such that $\sigma_{k-1} \leq t^{\prime} \leq \sigma_{k}$. Noticing that

$$
\left\|\widetilde{x}\left(t^{\prime}\right)-U\left(t^{\prime}\right)\right\| \leq\left\|\widetilde{x}\left(t^{\prime}\right)-\widetilde{x}\left(\sigma_{k}\right)\right\|+\left\|\widetilde{x}\left(\sigma_{k}\right)-U\left(\sigma_{k}\right)\right\|+\left\|U\left(\sigma_{k}\right)-U\left(t^{\prime}\right)\right\|
$$

we will evaluate the three components of the right-hand side separately.

Since $\widetilde{x}$ is affine on $\left[\sigma_{k}, \sigma_{k+1}\right]$, applying Proposition 3.11 gives

$$
\begin{aligned}
\left\|\widetilde{x}\left(t^{\prime}\right)-\widetilde{x}\left(\sigma_{k}\right)\right\| & \leq\left\|\widetilde{x}\left(\sigma_{k-1}\right)-\widetilde{x}\left(\sigma_{k}\right)\right\| \\
& =\left\|x_{k}-x_{k-1}\right\| \\
& \leq\left\|A\left(U_{0}\right)\right\| \sqrt{\left(\sigma_{k}-\sigma_{k-1}\right)^{2}+\tau_{k}+\tau_{k-1}} \\
& =\left\|A\left(U_{0}\right)\right\| \sqrt{2 \tau_{k}} .
\end{aligned}
$$

On another hand, Corollary 3.12 implies that

$$
\begin{aligned}
\left\|\widetilde{x}\left(\sigma_{k}\right)-U\left(\sigma_{k}\right)\right\| & =\left\|x_{k}-U\left(\sigma_{k}\right)\right\| \\
& \leq\left\|A\left(U_{0}\right)\right\| \sqrt{\tau_{k}} .
\end{aligned}
$$

Thirdly, using the mean value theorem as well as Corollary 3.6,

$$
\begin{aligned}
\left\|U\left(\sigma_{k}\right)-U\left(t^{\prime}\right)\right\| & \leq\left|\sigma_{k}-t^{\prime}\right| \max _{t^{\prime \prime} \in\left[t^{\prime}, \sigma_{k}\right]}\left\|U^{\prime}\left(t^{\prime \prime}\right)\right\| \\
& \leq\left|\sigma_{k}-\sigma_{k-1}\right| \cdot\left\|U^{\prime}(0)\right\| \\
& =\lambda_{k}\left\|A\left(U_{0}\right)\right\| .
\end{aligned}
$$

Adding inequalities (3.12) to (3.14) we thus deduce that

$$
\left\|\widetilde{x}\left(t^{\prime}\right)-U\left(t^{\prime}\right)\right\| \leq\left\|A\left(U_{0}\right)\right\|\left(\lambda_{k}+(1+\sqrt{2}) \sqrt{\tau_{k}}\right) .
$$

We use the facts that $\lambda_{k} \leq \sqrt{\lambda_{k}} \leq \sqrt{\max _{1 \leq i \leq n}\left\{\lambda_{i}\right\}}$, and that $\tau_{k} \leq \tau_{n} \leq t \max _{1 \leq i \leq n}\left\{\lambda_{i}\right\}$ to conclude the proof. 
This proposition has an interpretation in the particular framework of Example 3.2: consider a game with an expected duration of $t$. The previous result establishes that this game has a non normalized value close to $U(t)$, providing that at each stage the probability of playing is small (that is to say, if there is a high variance in the number of stages really played).

\section{Dynamical systems linked to the family $\Phi(\lambda, \cdot)$}

Let $\boldsymbol{\lambda}: \mathbf{R} \rightarrow$ ]0,1] be a continuous function. In this section we study the asymptotic behavior of the solution to evolution equation (2.18):

$$
u(t)+u^{\prime}(t)=\Phi(\boldsymbol{\lambda}(t), u(t)) \quad \text { with } u(0)=u_{0}
$$

where $\Phi$ is the operator defined by equation (2.7).

Remark 4.1. Since the mapping $(x, t) \rightarrow \Phi(\boldsymbol{\lambda}(t), x)-x$ is globally 2-Lipschitz in its first variable, CauchyLipschitz-Picard theorem ensures the existence and uniqueness of the solution of (2.18), and that it is defined on the whole set $\mathbb{R}^{+}$.

When the recession function $\Phi(0, \cdot)$ exists, any accumulation point $v$ of $v_{n}$ or $v_{\lambda}$ will satisfy

$$
\Phi(0, v)=v
$$

but equation (4.1) may have many solutions (for example in the case of games with incomplete information [24] any convex/concave function satisfies (4.1)). The evolution equation (2.18) may thus be seen as a perturbation of (4.1), and we will study the effect of some perturbations on the asymptotic behavior of the solution of (2.18). See for example [1] for a similar approach in the framework of convex minimization.

The main results of this section are the following:

- When $\boldsymbol{\lambda}$ is the constant $\lambda$, the solution of (2.18) converges to $v_{\lambda}$.

- When $\boldsymbol{\lambda}(t) \sim \frac{1}{t}$, the solution of (2.18) behave asymptotically as the family $\left\{v_{n}\right\}$.

- When $\boldsymbol{\lambda}(t)$ converges to 0 slowly enough, the solution of (2.18) behave asymptotically as the family $\left\{v_{\lambda}\right\}$.

The first two results are not surprising since in those cases evolution equation (2.18) is a continuous version of equation (2.11) or (2.9) respectively. The third result is of a different nature but is also natural: denote by $u_{\lambda}$ the solution of (2.18) when $\boldsymbol{\lambda}$ is the constant $\lambda$. We establish that if the parameterization $\boldsymbol{\lambda}$ in (2.18) is of slow variation, the solution $u$ evaluated at time $t$ is close to $u_{\lambda(t)}(t)$, hence to $v_{\lambda(t)}$ (see Fig. 1).

In the process of proving those three results, we also answer natural questions about the behavior of the solution $u$ of equation (2.18) as a function of the parameters, namely we will prove that:

- If $\boldsymbol{\lambda} \notin \ell^{1}$ the asymptotic behaviour of $u$ does not depend on the initial value $u_{0}$.

- If two parameterizations $\boldsymbol{\lambda}$ and $\widetilde{\boldsymbol{\lambda}}$ are asymptotically close, then it is also the case for the corresponding solutions $u$ and $\widetilde{u}$.

First we prove a simple fact that will be repeatedly used in the remaining of the paper. Recall, by equation (2.10), that for any $t \geq 0, v_{\boldsymbol{\lambda}(t)}$ is the only solution of

$$
v_{\boldsymbol{\lambda}(t)}=\Phi\left(\boldsymbol{\lambda}(t), v_{\boldsymbol{\lambda}(t)}\right)
$$

The following lemma relates the behavior of $u^{\prime}(t)$ to that of $u(t)-v_{\boldsymbol{\lambda}(t)}$ :

Lemma 4.2. Let $u$ be the solution of evolution equation (2.18) and $v_{\boldsymbol{\lambda}(\cdot)}$ be defined by (4.2). Then for any $t \geq 0,\left\|u(t)-v_{\boldsymbol{\lambda}(t)}\right\| \leq \frac{\left\|u^{\prime}(t)\right\|}{\boldsymbol{\lambda}(t)}$. 


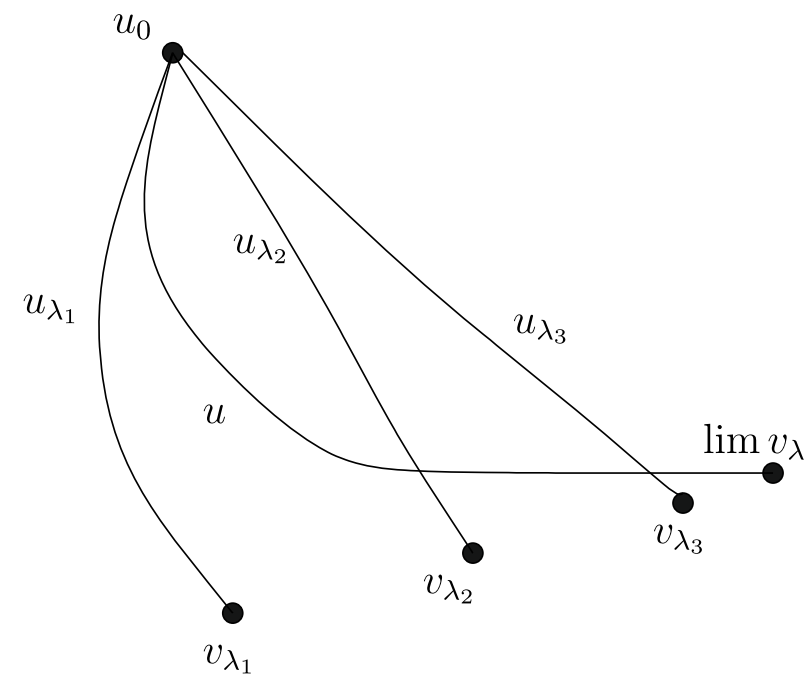

FIGURE 1. Trajectories of (2.18) for some constant parametrizations and for a slow parametrization.

Proof.

$$
\begin{aligned}
\left\|u^{\prime}(t)\right\| & =\|u(t)-\Phi(\boldsymbol{\lambda}(t), u(t))\| \\
& \geq\left\|u(t)-v_{\boldsymbol{\lambda}(t)}\right\|-\left\|\Phi(\boldsymbol{\lambda}(t), u(t))-\Phi\left(\boldsymbol{\lambda}(t), v_{\boldsymbol{\lambda}(t)}\right)\right\| \\
& \geq\left\|u(t)-v_{\boldsymbol{\lambda}(t)}\right\|-(1-\boldsymbol{\lambda}(t))\left\|u(t)-v_{\boldsymbol{\lambda}(t)}\right\| \\
& =\boldsymbol{\lambda}(t)\left\|u(t)-v_{\boldsymbol{\lambda}(t)}\right\| .
\end{aligned}
$$

\subsection{Constant case}

We start by considering the simplest case where the function $\boldsymbol{\lambda}$ is a constant $\lambda$. Equation (2.18) is then a continuous analogous of equation (2.11), so one can expect that $u(t)$ converges to $v_{\lambda}$, and indeed this is the case.

Start by a technical lemma:

Lemma 4.3. If $f$ satisfies $f(t)+f^{\prime}(t)=B(f(t))$, where $B$ is an $1-\lambda$ contracting operator, then

$$
\left\|f^{\prime}(t)\right\| \leq\left\|f^{\prime}(0)\right\| \cdot \mathrm{e}^{-\lambda t} .
$$

Proof. Let $h>0$ and $f_{h}(t)=\frac{f(t+h)-f(t)}{h}$. Since $B$ is $(1-\lambda)$ contracting:

$$
\begin{aligned}
\left\|f_{h}(t)+f_{h}^{\prime}(t)\right\| & =\frac{1}{h}\left\|f(t+h)+f^{\prime}(t+h)-\left[f(t)+f^{\prime}(t)\right]\right\| \\
& =\frac{1}{h}\|B(f(t+h))-B(f(t))\| \\
& \leq(1-\lambda)\left\|f_{h}(t)\right\| .
\end{aligned}
$$

Proposition 3.4 applied to $f_{h}$ thus implies that

$$
\left\|f_{h}(t)\right\| \leq\left\|f_{h}(0)\right\| \cdot \mathrm{e}^{-\lambda t}
$$

and letting $h$ go to 0 gives the result. 
An immediate consequence is:

Corollary 4.4. If $u$ is the solution of (2.18) with $\boldsymbol{\lambda}(t):=\lambda$, then

$$
\lim _{t \rightarrow+\infty} u(t)=v_{\lambda}
$$

Proof. Lemmas 4.2 and 4.3 imply that

$$
\left\|u(t)-v_{\lambda}\right\|=\left\|u(t)-v_{\boldsymbol{\lambda}(t)}\right\| \leq \frac{\left\|u^{\prime}(t)\right\|}{\lambda(t)} \leq\left\|u^{\prime}(0)\right\| \cdot \frac{\mathrm{e}^{-\lambda t}}{\lambda}
$$

and the right member goes to 0 as $t$ tends to $+\infty$.

\subsection{Some generalities on the non-autonomous case}

The case when the parameterization $\boldsymbol{\lambda}$ is not constant is more difficult to handle: the same method as in the proof of Corollary 4.4 leads to

$$
u(t+h)-u(t)+u^{\prime}(t+h)-u^{\prime}(t)=\Phi(\boldsymbol{\lambda}(t+h), u(t+h))-\Phi(\boldsymbol{\lambda}(t), u(t))
$$

but Proposition 3.4 does not apply.

However, we can prove if the perturbation is strong enough:

Proposition 4.5. If $\int_{0}^{+\infty} \boldsymbol{\lambda}(t) \mathrm{d} t=+\infty$, the asymptotic behavior of $u$ solution of (2.18) does not depend of the choice of $u(0)$.

Proof. Let $u$ and $v$ be two solutions of (2.18), define the function $g$ by $g(x)=\|u(x)-v(x)\|$. According to Proposition 3.4,

from which the proposition follows.

$$
g(x) \leq g(0) \cdot \mathrm{e}^{-\int_{0}^{x} \boldsymbol{\lambda}(t) \mathrm{d} t}
$$

\subsection{Case of $\lambda(t) \simeq \frac{1}{t}$}

When $\boldsymbol{\lambda}(t)=\frac{1}{t}$, equation (2.18) is the continuous counterpart of equation (2.9), so we expect $u(t)$ to have the same asymptotic behavior as $v_{n}$. This will be proved with an additional hypothesis on $\Phi$ in the next section. Here we show a slightly weaker result without any assumption.

Proposition 4.6. There exists a function $\boldsymbol{\lambda}:[0,+\infty] \rightarrow] 0,1]$ such that $\boldsymbol{\lambda}(t) \sim \frac{1}{t}$ and for which the solution $w$ of (2.18) satisfies

$$
\left\|w(n)-v_{n}\right\| \underset{n \rightarrow+\infty}{\longrightarrow} 0
$$

Proof. Let $U$ be the solution of (2.15) and $v(t)=\frac{U(t)}{t+1}$, which thus satisfies

$$
(t+2) v(t)+(t+1) v^{\prime}(t)=J((t+1) v(t))
$$

Define $\zeta(t)=t+\ln (1+t)$. By making the change of time $s=\zeta(t)$ and $w(s)=v(t)$, we get

$$
w(s)+w^{\prime}(s)=\Phi\left(\frac{1}{2+\zeta^{-1}(s)}, w(s)\right)
$$

and $w$ is thus solution of $(2.18)$ with

$$
\lambda(t)=\frac{1}{2+\zeta^{-1}(t)}=\frac{1}{t}+\frac{\ln (t)}{t^{2}}+o\left(\frac{\ln (t)}{t^{2}}\right) .
$$


Moreover,

$$
\left\|w(n)-v_{n}\right\| \leq\left\|v(n)-v_{n}\right\|+\left\|v\left(\zeta^{-1}(n)\right)-v(n)\right\| .
$$

We already know by Corollary 3.8 that $\left\|v(n)-v_{n}\right\|$ goes to 0 as $n$ tends to $+\infty$. On the other hand, by the mean value theorem,

$$
\left\|v\left(\zeta^{-1}(n)\right)-v(n)\right\| \leq\left(n-\zeta^{-1}(n)\right) \cdot \max _{x \in\left[\zeta^{-1}(n), n\right]}\left\|v^{\prime}(x)\right\| .
$$

By definition of $v, v^{\prime}(x)=\frac{(x+1) U^{\prime}(x)-U(x)}{(x+1)^{2}}$ hence Corollary 3.6 implies that

$$
\begin{aligned}
\left\|v^{\prime}(x)\right\| & \leq \frac{(x+1)\left\|U^{\prime}(x)\right\|+\|U(x)\|}{(x+1)^{2}} \\
& \leq \frac{(x+1)\left\|U^{\prime}(0)\right\|+\|U(0)\|+x\left\|U^{\prime}(0)\right\|}{(x+1)^{2}} \\
& \leq \frac{C}{x+1}
\end{aligned}
$$

for $C=2 \max \left(\|U(0)\|,\left\|U^{\prime}(0)\right\|\right)$.

Replacing in equation (4.6) gives

$$
\left\|v\left(\zeta^{-1}(n)\right)-v(n)\right\| \leq C \frac{n-\zeta^{-1}(n)}{1+\zeta^{-1}(n)}
$$

which goes to 0 since $\zeta(n) \sim n$, and we have thus proved that

$$
\left\|w(n)-v_{n}\right\| \underset{n \rightarrow+\infty}{\longrightarrow} 0 .
$$

An interesting corollary of this proposition, which gives a sufficient condition for convergence of both $v_{n}$ and $v_{\lambda}$ to the same limit, is:

Corollary 4.7. Let $U$ be the solution of (2.15). If $U^{\prime}(t)$ converges to $l$ when $t$ goes to $+\infty$, then $v_{n}$ and $v_{\lambda}$ converge to $l$ as well as $n$ goes to $+\infty$ and $\lambda$ goes to 0 , respectively.

Proof. Suppose that $U^{\prime}(t)$ converges to $l$ when $t$ goes to $+\infty$. Then $v(t)=\frac{U(t)}{t}$ converges to $l$ as well, and so does $v_{n}$ according to Corollary 3.8 .

On the other hand,

$$
t v^{\prime}(t)=U^{\prime}(t)-\frac{U(t)}{t} \rightarrow l-l=0
$$

so $v^{\prime}(t)=o\left(\frac{1}{t}\right)$. Define $\zeta, \lambda$ and $w$ as in the proof of the preceding proposition; then $w(t)=v\left(\zeta^{-1}(t)\right)$ converges also to $l$ and by definition

$$
w^{\prime}(\zeta(t))=\frac{t+1}{t+2} v^{\prime}(t)=o\left(\frac{1}{t}\right)
$$

Since $\zeta(t) \sim t$ and $\boldsymbol{\lambda}(t) \sim \frac{1}{t}$ this implies that $\frac{\left\|w^{\prime}(t)\right\|}{\boldsymbol{\lambda}(t)}=o(1)$. According to Lemma 4.2, this implies that $\left\|w(t)-v_{\boldsymbol{\lambda}(t)}\right\|=o(1)$, and so $v_{\lambda}$ tends to $l$ as $\lambda$ goes to 0 . 


\subsection{Case of a slow parameterization}

From now on the following assumption $(\mathcal{H})$ will be made: there is a constant $C$ such that

$$
\|\Phi(\lambda, x)-\Phi(\mu, x)\| \leq|\lambda-\mu|(C+\|x\|) \quad \forall x \in X \quad \forall(\lambda, \mu) \in] 0,1]^{2} .
$$

Remark 4.8. $(\mathcal{H})$ is satisfied as soon as $J$ is the Shapley operator $(2.12)$ of a game with bounded payoff since in that case

$$
\|\Phi(\lambda, x)-\Phi(\mu, x)\|_{\infty} \leq|\lambda-\mu|\left(\|g\|_{\infty}+\|x\|_{\infty}\right) .
$$

Remark 4.9. Hypothesis $(\mathcal{H})$ implies that for every $\lambda$ and $\mu$

$$
\frac{\left\|v_{\lambda}-v_{\mu}\right\|}{|\lambda-\mu|} \leq \frac{C^{\prime}}{\lambda}
$$

for some constant $C^{\prime}$ : in some sense $(\mathcal{H})$ is thus a statement about the speed of variation of the family $\left\{v_{\lambda}\right\}$.

The principal result of this subsection is Corollary 4.12 which states that under this hypothesis, if the parameterization $\boldsymbol{\lambda}$ converges slowly enough to 0 , then the corresponding solution of (2.18) has the same asymptotic behavior as the family $\left\{v_{\lambda}\right\}$. We start by a technical result:

Proposition 4.10. Let $\boldsymbol{\lambda}$ be a $\mathcal{C}^{1}$ function from $[0,+\infty[$ to $] 0,1]$ and let $L: \mathbb{R}^{+} \rightarrow \mathbb{R}$ be defined by $L(t)=\mathrm{e}^{\int_{0}^{t}\left[\frac{\left|\boldsymbol{\lambda}^{\prime}(s)\right|}{\lambda(s)}-\boldsymbol{\lambda}(s)\right] \mathrm{d} s}$. Then the corresponding solution $u$ of (2.18) satisfies:

$$
\left\|u(t)-v_{\boldsymbol{\lambda}(t)}\right\| \leq \frac{L(t)}{\boldsymbol{\lambda}(t)}\left[\left\|u^{\prime}(0)\right\|+\left(C+C^{\prime}\right) \int_{0}^{t} \frac{\left|\boldsymbol{\lambda}^{\prime}(s)\right|}{L(s)} \mathrm{d} s\right]
$$

where $C$ is the constant in condition $(\mathcal{H})$ and $C^{\prime}=\sup _{\lambda \in] 0,1]}\left\|v_{\lambda}\right\|$.

Proof. For any $h>0$, define $u_{h}(t)=\frac{u(t+h)-u(t)}{h}$ and $\boldsymbol{\lambda}_{h}(t)=\frac{\boldsymbol{\lambda}(t+h)-\boldsymbol{\lambda}(t)}{h}$. Since $u$ is $\mathcal{C}^{1}$,

$$
u_{h}(t)=u^{\prime}(t)+\frac{1}{h} \int_{t}^{t+h} u^{\prime}(s)-u^{\prime}(t) \mathrm{d} s
$$

which implies, by uniform continuity of $u$ on any compact set, that the restriction of $u_{h}$ to any closed interval converges uniformly to $u^{\prime}$ as $h$ goes to 0 . Similarly, the restriction of $\boldsymbol{\lambda}_{h}$ to any closed interval converges uniformly to $\boldsymbol{\lambda}^{\prime}$ as $h$ goes to 0 .

Since $u$ satisfies equation (2.18), for any $h$ and $t$,

$$
\begin{aligned}
\left\|u_{h}(t)+u_{h}^{\prime}(t)\right\| & =\frac{1}{h}\|\Phi(\boldsymbol{\lambda}(t+h), u(t+h))-\Phi(\boldsymbol{\lambda}(t), u(t))\| \\
& \leq \frac{1}{h}\|\Phi(\boldsymbol{\lambda}(t+h), u(t+h))-\Phi(\boldsymbol{\lambda}(t+h), u(t))\|+\frac{1}{h}\|\Phi(\boldsymbol{\lambda}(t+h), u(t))-\Phi(\boldsymbol{\lambda}(t)(t), u(t))\| \\
& \leq(1-\boldsymbol{\lambda}(t+h))\left\|u_{h}(t)\right\|+\left|\boldsymbol{\lambda}_{h}(t)\right|(C+\|u(t)\|),
\end{aligned}
$$

by hypothesis $(\mathcal{H})$. According to Lemma 4.2 , this implies that

$$
\left\|u_{h}(t)+u_{h}^{\prime}(t)\right\| \leq(1-\boldsymbol{\lambda}(t+h))\left\|u_{h}(t)\right\|+\left|\boldsymbol{\lambda}_{h}(t)\right|\left(C+C^{\prime}+\frac{\left\|u^{\prime}(t)\right\|}{\boldsymbol{\lambda}(t)}\right)
$$

where $C^{\prime}$ is a majorant of the family $\left\|v_{\lambda}\right\|$. 
Fix $t_{0}>0$, and let $\varepsilon>0$. Since $\boldsymbol{\lambda}(t)$ is bounded from below on $\left[0, t_{0}\right]$ and using the uniform convergence of $u_{h}$ to $u^{\prime}$ on $\left[0, t_{0}\right]$, one obtains that for $h$ small enough, and for every $t \leq t_{0}$,

$$
\left\|u_{h}(t)+u_{h}^{\prime}(t)\right\| \leq\left(1-\boldsymbol{\lambda}(t+h)+\frac{\left|\boldsymbol{\lambda}_{h}(t)\right|}{\boldsymbol{\lambda}(t)}\right)\left\|u_{h}(t)\right\|+\left(C+C^{\prime}+\varepsilon\right)\left|\boldsymbol{\lambda}_{h}(t)\right| .
$$

Then applying Proposition 3.4 to $u_{h}$ implies that for any $h$ small enough and $t \leq t_{0}$,

$$
\left\|u_{h}(t)\right\| \leq \mathrm{e}^{\int_{0}^{t}\left[\frac{\left|\boldsymbol{\lambda}_{h}(s)\right|}{\boldsymbol{\lambda}(s)}-\boldsymbol{\lambda}(s+h)\right] \mathrm{d} s}\left(\left\|u_{h}(0)\right\|+\left(C+C^{\prime}+\varepsilon\right) \int_{0}^{t}\left|\boldsymbol{\lambda}_{h}(s)\right| \mathrm{e}^{\int_{0}^{s}\left[\boldsymbol{\lambda}(r+h)-\frac{\left|\boldsymbol{\lambda}_{h}(r)\right|}{\boldsymbol{\lambda}(r)}\right] \mathrm{d} r} \mathrm{~d} s\right) .
$$

Using the uniform convergence of $\boldsymbol{\lambda}_{h}$ and $\boldsymbol{\lambda}(\cdot+h)$ on $\left[0, t_{0}\right]$, letting $h$ go to 0 implies that for any $t \leq t_{0}$,

$$
\left\|u^{\prime}(t)\right\| \leq L(t)\left(\left\|u^{\prime}(0)\right\|+\left(C+C^{\prime}+\varepsilon\right) \int_{0}^{t} \frac{\left|\boldsymbol{\lambda}^{\prime}(s)\right|}{L(s)} \mathrm{d} s\right) .
$$

Since this is true for every $t_{0}$ and $\varepsilon$, using Lemma 4.2 again gives

$$
\left\|u(t)-v_{\boldsymbol{\lambda}(t)}\right\| \leq \frac{L(t)}{\boldsymbol{\lambda}(t)}\left[\left\|u^{\prime}(0)\right\|+\left(C+C^{\prime}\right) \int_{0}^{t} \frac{\left|\boldsymbol{\lambda}^{\prime}(s)\right|}{L(s)} \mathrm{d} s\right] .
$$

Remark 4.11. If in Proposition 4.10 we suppose in addition that $\boldsymbol{\lambda}$ is nonincreasing, we get the simpler inequality

$$
\left\|u(t)-v_{\boldsymbol{\lambda}(t)}\right\| \leq \frac{\mathrm{e}^{-\int_{0}^{t} \boldsymbol{\lambda}(s) \mathrm{d} s}}{\boldsymbol{\lambda}^{2}(t)}\left[\left\|u^{\prime}(0)\right\|-\left(C+C^{\prime}\right) \int_{0}^{t} \boldsymbol{\lambda}(s) \boldsymbol{\lambda}^{\prime}(s) \mathrm{e}^{\int_{0}^{s} \boldsymbol{\lambda}(r) \mathrm{d} r} \mathrm{~d} s\right] .
$$

As a corollary to Proposition 4.10 we can now prove:

Corollary 4.12. Let $\boldsymbol{\lambda}$ be a $\mathcal{C}^{1}$ function from $[0,+\infty[$ to $] 0,1]$, such that $\frac{\boldsymbol{\lambda}^{\prime}(t)}{\boldsymbol{\lambda}^{2}(t)}$ converges to 0 as $t$ goes to $+\infty$, and let $u$ be the corresponding solution of equation (2.18). Then $\left\|u(t)-v_{\boldsymbol{\lambda}(t)}\right\|$ goes to 0 as $t$ goes to $+\infty$.

Proof. First notice that $\left(\frac{1}{\lambda(t)}\right)^{\prime}=o(1)$, so $\frac{1}{\lambda(t)}=o(t)$ which implies that $\boldsymbol{\lambda}(t) \notin \ell^{1}$.

Next we prove that

$$
\frac{L(t)}{\boldsymbol{\lambda}(t)}=o(1)
$$

Since the left-hand side is equal to $\frac{\mathrm{e}^{\int_{0}^{t}\left[\frac{\left|\lambda^{\prime}(s)\right|}{\lambda(s)}-\frac{\lambda^{\prime}(s)}{\lambda(s)}-\boldsymbol{\lambda}(s)\right] \mathrm{d} s}}{\boldsymbol{\lambda}(0)}$, the result is deduced from the fact that $\frac{\boldsymbol{\lambda}^{\prime}(s)}{\boldsymbol{\lambda}(s)}=$ $o(\boldsymbol{\lambda}(s))$ and that $\boldsymbol{\lambda}(t) \notin \ell^{1}$.

Finally we prove that

$$
\int_{0}^{t} \frac{\left|\boldsymbol{\lambda}^{\prime}(s)\right|}{L(s)} \mathrm{d} s=o\left(\frac{\boldsymbol{\lambda}(t)}{L(t)}\right)
$$

Since the right-hand side diverges to $+\infty$, it is enough to prove that the derivative satisfies

$$
\frac{\left|\boldsymbol{\lambda}^{\prime}(t)\right|}{L(t)}=o\left(\frac{\boldsymbol{\lambda}^{\prime}(t)+\boldsymbol{\lambda}^{2}(t)-\left|\boldsymbol{\lambda}^{\prime}(t)\right|}{L(t)}\right)
$$

which is true since $\boldsymbol{\lambda}^{\prime}(t)=o\left(\boldsymbol{\lambda}^{2}(t)\right)$. 
Remark 4.13. Note the similarity of this proposition with some approximation results for dynamical systems in the framework of Hilbert spaces, for example the slow parameterization in [1]:

- First there is a parallel between the strong monotonicity condition in [1], p. 523, and our assumption that the $\Phi(\lambda, \cdot)$ are contracting.

- Second between a condition about the derivative of the trajectory in the same paper, p. 528, and our hypothesis $(\mathcal{H})$ (see Rem. 4.9).

- Third the slow-convergence condition is the same (see condition (ii) in [1], p. 528).

- Lastly, results of both papers are of the same nature: convergence of a certain family $\left(\left\{v_{\lambda}\right\}\right.$ in this paper) implies that the solution of any slowly-perturbed evolution equation tends to this limit as time goes to infinity.

A difference however is the fact that in this paper we also have a reciprocal: if for any slow parameterization $\boldsymbol{\lambda}$ the solution $u(t)$ of (2.18) converges as $t$ goes to infinity, then the family $v_{\lambda}$ converges to the same limit as $\lambda$ goes to 0 .

Remark 4.14. In the proof of Proposition 4.10 only the three following hypotheses on the family $\Phi$ were used:

(i) $\Phi(\cdot, x)$ satisfies condition $\mathcal{H}$ for all $x$.

(ii) $\Phi(\lambda, \cdot)$ is $1-\lambda$ contracting for every $\lambda \in] 0,1]$.

(iii) The fixed points $v_{\lambda}$ are uniformly bounded.

The two last ones are satisfied as soon as $\Phi(\lambda, x)=\lambda J\left(\frac{1-\lambda}{\lambda} x\right)$ for a nonexpansive operator $J$, but this is not a necessary condition for Proposition 4.10 to hold.

Remark 4.15. In fact, the more general result holds: suppose that the family $\Phi$ satisfies the three hypotheses:

(i) There exists a constant $C$ and a continuous function $M$ from $] 0,1]$ to $\mathbb{R}^{+}$such that for any $(x, \lambda, \mu)$ in $X \times] 0,1]^{2}$

$$
\|\Phi(\lambda, x)-\Phi(\mu, x)\| \leq\left|\int_{\lambda}^{\mu} M(\gamma) \mathrm{d} \gamma\right|(C+\|x\|)
$$

(ii) There exists a continuous function $\beta:] 0,1] \rightarrow] 0,1]$ such that $\Phi(\lambda, \cdot)$ is $1-\beta(\lambda)$ contracting.

(iii) The fixed points $v_{\lambda}$ of $\Phi(\lambda, \cdot)$ are uniformly bounded by $C^{\prime}$.

Let $\boldsymbol{\lambda}$ be a $\mathcal{C}^{1}$ function from $[0,+\infty[$ to $] 0,1]$. Then the corresponding solution $u$ of (2.18) satisfies

$$
\begin{aligned}
\left\|u(t)-v_{\boldsymbol{\lambda}(t)}\right\| \leq & \frac{\mathrm{e}_{0}^{t} \frac{\left|\boldsymbol{\lambda}^{\prime}(s)\right| M(\boldsymbol{\lambda}(s))}{\beta(\boldsymbol{\lambda}(s))}-\beta(\boldsymbol{\lambda}(s)) \mathrm{d} s}{\beta(\boldsymbol{\lambda}(t))} \\
& \times\left[\left\|u^{\prime}(0)\right\|+\left(C+C^{\prime}\right) \int_{0}^{t}\left|\boldsymbol{\lambda}^{\prime}(s)\right| M(\boldsymbol{\lambda}(s)) \mathrm{e}^{\int_{0}^{s}\left[\beta(\boldsymbol{\lambda}(i))-\frac{\left|\boldsymbol{\lambda}^{\prime}(i)\right| M(\boldsymbol{\lambda}(i))}{\beta(\boldsymbol{\lambda}(i))}\right] \mathrm{d} i} \mathrm{~d} s\right] .
\end{aligned}
$$

This implies that $\left\|u(t)-v_{\boldsymbol{\lambda}(t)}\right\|$ tends to 0 as soon as $\beta$ is $\mathcal{C}^{1}$ and the parameterization $\lambda$ satisfies both properties :

$$
\begin{aligned}
& \text { (iv) } \frac{\boldsymbol{\lambda}^{\prime}(t) M(\boldsymbol{\lambda}(t))}{\beta^{2}(\boldsymbol{\lambda}(t))}=o(1) ; \\
& \text { (v) } \frac{\boldsymbol{\lambda}^{\prime}(t) \beta^{\prime}(\boldsymbol{\lambda}(t))}{\beta^{2}(\boldsymbol{\lambda}(t))}=o(1) .
\end{aligned}
$$

Notice again the similarity with [1].

Another interesting consequence of hypothesis $(\mathcal{H})$ is Corollary 4.17 which states that if two parameterizations are close to one other, then this is also the case for the trajectories. We first prove a technical result using the same approach as in the proof of Proposition 4.10:

Proposition 4.16. Let $u$ and $v$ be the two solutions of (2.18) for some functions $\boldsymbol{\lambda}$ and $\boldsymbol{\mu}$ respectively. Then for any $t \geq 0$,

$$
\|u(t)-v(t)\| \leq \mathrm{e}^{-\int_{0}^{t} \boldsymbol{\mu}(s) \mathrm{d} s}\left(\left\|u_{0}-v_{0}\right\|+\int_{0}^{t}(C+\|u(s)\|)|\boldsymbol{\lambda}(s)-\boldsymbol{\mu}(s)| \cdot \mathrm{e}^{\int_{0}^{s} \boldsymbol{\mu}(i) \mathrm{d} i} \mathrm{~d} s\right) .
$$


Proof. Let $f=u-v$, then

$$
\begin{aligned}
\left\|f(t)+f^{\prime}(t)\right\| & =\|\Phi(\boldsymbol{\lambda}(t), u(t))-\Phi(\boldsymbol{\mu}(t), v(t))\| \\
& \leq\|\Phi(\boldsymbol{\lambda}(t), u(t))-\Phi(\boldsymbol{\mu}(t), u(t))\|+\|\Phi(\boldsymbol{\mu}(t), u(t))-\Phi(\boldsymbol{\mu}(t), v(t))\| \\
& \leq|\boldsymbol{\lambda}(t)-\boldsymbol{\mu}(t)| \cdot(C+\|u(t)\|)+(1-\boldsymbol{\mu}(t)) \cdot\|f(t)\|
\end{aligned}
$$

because of hypothesis $(\mathcal{H})$ and contraction of $\Phi(\lambda, \cdot)$. Applying Proposition 3.4 gives the result.

In particular one has:

Corollary 4.17. Let $u$ and $v$ the two solutions of (2.18) for some functions $\boldsymbol{\lambda}$ and $\boldsymbol{\mu}$ respectively. Assume that $u$ is bounded and $\boldsymbol{\mu} \notin \ell^{1}$, then $\|u(t)-v(t)\| \rightarrow 0$ in the two following cases:

(a) $\boldsymbol{\mu}(t) \sim \boldsymbol{\lambda}(t)$ as $t$ goes to $+\infty$;

(b) $|\boldsymbol{\lambda}-\boldsymbol{\mu}| \in \ell^{1}$.

Proof. Let $L$ be a bound for $u$. By the preceding proposition we know that

$$
\|u(t)-v(t)\| \leq \mathrm{e}^{-\int_{0}^{t} \boldsymbol{\mu}(s) \mathrm{d} s}\left(\left\|u_{0}-v_{0}\right\|+(C+L) \int_{0}^{t}|\boldsymbol{\lambda}(s)-\boldsymbol{\mu}(s)| \cdot \mathrm{e}^{\int_{0}^{s} \boldsymbol{\mu}(i) \mathrm{d} i} \mathrm{~d} s\right)
$$

so it suffices to show that

$$
\int_{0}^{t}|\boldsymbol{\lambda}(s)-\boldsymbol{\mu}(s)| \cdot \mathrm{e}^{\int_{0}^{s} \boldsymbol{\mu}(i) \mathrm{d} i} \mathrm{~d} s=o\left(\mathrm{e}^{\int_{0}^{t} \boldsymbol{\mu}(s) \mathrm{d} s}\right) .
$$

(a) Assume that $\boldsymbol{\mu}(t) \sim \boldsymbol{\lambda}(t)$, that is $\frac{|\boldsymbol{\lambda}(t)-\boldsymbol{\mu}(t)|}{\boldsymbol{\mu}(t)}=o(1)$. This implies that

$$
|\boldsymbol{\lambda}(t)-\boldsymbol{\mu}(t)| \cdot \mathrm{e}^{\int_{0}^{t} \boldsymbol{\mu}(s) \mathrm{d} s}=o\left(\boldsymbol{\mu}(t) \mathrm{e}^{\int_{0}^{t} \boldsymbol{\mu}(s) \mathrm{d} s}\right)
$$

which gives the result by integrating.

(b) Assume that $|\boldsymbol{\lambda}-\boldsymbol{\mu}| \in \ell^{1}$, let $\varepsilon>0$ and $T$ such that $\int_{T}^{+\infty}|\boldsymbol{\lambda}(s)-\boldsymbol{\mu}(s)| \mathrm{d} s<\varepsilon$. Then for $t>T$,

$$
\begin{aligned}
I & :=\int_{0}^{t}|\boldsymbol{\lambda}(s)-\boldsymbol{\mu}(s)| \cdot \mathrm{e}^{\int_{0}^{s} \boldsymbol{\mu}(i) \mathrm{d} i} \mathrm{~d} s \\
& =\int_{0}^{T}|\boldsymbol{\lambda}(s)-\boldsymbol{\mu}(s)| \cdot \mathrm{e}^{\int_{0}^{s} \boldsymbol{\mu}(i) \mathrm{d} i} \mathrm{~d} s+\int_{T}^{t}|\boldsymbol{\lambda}(s)-\boldsymbol{\mu}(s)| \cdot \mathrm{e}^{\int_{0}^{s} \boldsymbol{\mu}(i) \mathrm{d} i} \mathrm{~d} s \\
& \leq \mathrm{e}^{\int_{0}^{T} \boldsymbol{\mu}(s) \mathrm{d} s} \int_{0}^{T}|\boldsymbol{\lambda}(s)-\boldsymbol{\mu}(s)| \mathrm{d} s+\mathrm{e}^{\int_{0}^{t} \boldsymbol{\mu}(s) \mathrm{d} s} \int_{T}^{t}|\boldsymbol{\lambda}(s)-\boldsymbol{\mu}(s)| \mathrm{d} s \\
& \leq \mathrm{e}^{\int_{0}^{T} \boldsymbol{\mu}(s) \mathrm{d} s} \int_{0}^{T}|\boldsymbol{\lambda}(s)-\boldsymbol{\mu}(s)| \mathrm{d} s+\varepsilon \mathrm{e}^{\int_{0}^{t} \boldsymbol{\mu}(s) \mathrm{d} s} \\
& \leq 2 \varepsilon \mathrm{e}_{0}^{\int_{0}^{t} \boldsymbol{\mu}(s) \mathrm{d} s}
\end{aligned}
$$

for all $t$ large enough since $\mathrm{e}^{\int_{0}^{t} \boldsymbol{\mu}(s) \mathrm{d} s}$ diverges to $+\infty$ as $t$ goes to $+\infty$.

Some interesting corollaries follows immediately: first because of Corollary 4.4, we get:

Corollary 4.18. If $\boldsymbol{\lambda}(t) \rightarrow \lambda>0$, then $u(t) \rightarrow v_{\lambda}$. 
Then, combining the results of Section 4.3 and Corollaries 4.12 and 4.17 we deduce the following corollary bringing to light the tight difference between dynamics related to $\lim v_{n}$ and $\lim v_{\lambda}$ :

Corollary 4.19. For $\alpha \in\left[0,1\left[\right.\right.$, let $u^{\alpha}$ be the solution of

$$
u(t)+u^{\prime}(t)=\Phi\left((1+t)^{\alpha-1}, u(t)\right) \quad \text { with } u(0)=u_{0}
$$

Then $u^{0}(t)$ converges to some $l \in X$ when $t$ goes to $+\infty$ iff $v_{n}$ converges to $l$ as $n$ goes to $+\infty$; and for $\left.\alpha \in\right] 0,1[$ $u^{\alpha}(t)$ converges to some $l \in X$ as $t$ goes to $+\infty$ iff $v_{\lambda}$ converges to $l$ as $\lambda$ goes to 0 .

\subsection{Back to discrete time}

We proved in the last section that under hypothesis $(\mathcal{H})$, the solution of $(2.18)$ has the same asymptotic behavior as the family $\left\{v_{\lambda}\right\}$ as soon as $\boldsymbol{\lambda}$ converges slowly enough to 0 . One may wonder if it is true as well in discrete time. For any sequence $\left(\lambda_{n}\right)_{n \in \mathbb{N}}$ in $\left.] 0,1\right]$, define the discrete counterpart of equation (2.18) :

$$
w_{n}=\Phi\left(\lambda_{n}, w_{n-1}\right) \quad \text { with } w(0)=w_{0} .
$$

Then one obtains the discrete version of Corollary 4.12 :

Proposition 4.20. Let $\lambda_{n}$ be a sequence in ]0,1]. Assume that both $\lambda_{n}$ and $\frac{1}{\lambda_{n}}-\frac{1}{\lambda_{n+1}}$ tend to 0 as $n$ goes to $+\infty$. Then the solution $\left(w_{n}\right)_{n \in \mathbb{N}}$ of (4.15) satisfies

$$
\left\|v_{\lambda_{n}}-w_{n}\right\| \rightarrow 0
$$

as $n$ goes to $+\infty$.

Proof. The sequence $\gamma_{n}=\frac{1}{\lambda_{n}}$ tends to $+\infty$ and satisfies $\gamma_{n}-\gamma_{n-1} \rightarrow 0$ as $n$ goes to $+\infty$. This implies the existence of an interpolation function $\gamma: \mathbb{R} \rightarrow \mathbb{R}$ which is $\mathcal{C}^{2}$ and such that for all $n$ in $\mathbb{N}, \gamma(n)=\gamma_{n}$, $\lim _{+\infty} \gamma(t)=+\infty$ and $\lim _{+\infty} \gamma^{\prime}(t)=0$. The function $\boldsymbol{\lambda}:=\frac{1}{\gamma}$ thus satisfies $\boldsymbol{\lambda}(n)=\lambda_{n}$ and all the hypotheses of Corollary 4.12. Let us denote by $u$ the corresponding solution of equation (2.18). By Corollary 4.12 it is enough to show that $\left\|w_{n}-u(n)\right\| \rightarrow 0$ as $n$ goes to $+\infty$.

Define $a_{n}:=\left\|w_{n}-u(n)\right\|$ and let $\varepsilon>0$. Then

$$
\begin{aligned}
a_{n} & =\left\|\Phi\left(\lambda_{n}, w_{n-1}\right)-\Phi\left(\lambda_{n}, u(n)\right)+u^{\prime}(n)\right\| \\
& \leq\left(1-\lambda_{n}\right)\left\|w_{n-1}-u(n)\right\|+\left\|u^{\prime}(n)\right\| \\
& \leq\left(1-\lambda_{n}\right)\left\|w_{n-1}-u(n-1)\right\|+\|u(n)-u(n-1)\|+\left\|u^{\prime}(n)\right\| \\
& \leq\left(1-\lambda_{n}\right) a_{n-1}+2 \sup _{t \in[n-1, n]}\left\|u^{\prime}(t)\right\| \\
& \leq\left(1-\lambda_{n}\right) a_{n-1}+2 \sup _{t \in[n-1, n]}\left\|\frac{u^{\prime}(t)}{\boldsymbol{\lambda}(t)}\right\| \cdot \sup _{t \in[n-1, n]} \boldsymbol{\lambda}(t) \\
& \leq\left(1-\lambda_{n}\right) a_{n-1}+2 \varepsilon \sup _{t \in[n-1, n]} \boldsymbol{\lambda}(t)
\end{aligned}
$$

for every $n$ large enough because of Corollary 4.12 . 
Denote $s_{n}=\max _{t \in[n-1, n]} \boldsymbol{\lambda}(t)=o(1)$, and choose $t_{n} \in[n-1, n]$ such that $s_{n}=\boldsymbol{\lambda}\left(t_{n}\right)$. Let $T>0$ such that $\left|\boldsymbol{\lambda}^{\prime}(t)\right| \leq \boldsymbol{\lambda}^{2}(t)$ for every $t \geq T$, then by the mean value theorem, for any $n \geq T+1$,

$$
\begin{aligned}
\left|s_{n}-\lambda_{n}\right| & =\left|\boldsymbol{\lambda}\left(t_{n}\right)-\boldsymbol{\lambda}(n)\right| \\
& \leq\left|t_{n}-n\right| \cdot \sup _{t \in[n-1, n]}\left|\boldsymbol{\lambda}^{\prime}(t)\right| \\
& \leq \sup _{t \in[n-1, n]} \boldsymbol{\lambda}^{2}(t) \\
& =s_{n}^{2} \\
& =o\left(s_{n}\right)
\end{aligned}
$$

so that $s_{n} \sim \lambda_{n}$ as $n$ goes to $+\infty$. Together with (4.16) this implies that there exists $N$ such that for all $n \geq N$,

$$
a_{n} \leq\left(1-\lambda_{n}\right) a_{n-1}+3 \varepsilon \lambda_{n}
$$

and so by induction one prove that for all $k \in \mathbb{N}$,

$$
a_{N+k}-3 \varepsilon \leq\left(a_{N}-3 \varepsilon\right) \prod_{i=1}^{k}\left(1-\lambda_{N+i}\right) .
$$

Now $\frac{1}{\lambda_{n}}-\frac{1}{\lambda_{n-1}} \rightarrow 0$ implies that $\frac{1}{n}=o\left(\lambda_{n}\right)$, so the product goes to 0 and we deduce that $a_{N+k} \leq 4 \varepsilon$ for $k$ large enough.

Corollary 4.21. $v_{\lambda}$ converges as $\lambda$ goes to 0 if and only if there exists a sequence $\lambda_{n}$ satisfying the hypothesis of Proposition 4.20 such that the corresponding sequence $w_{n}$ defined by (4.15) converges.

Proof. Let $\lambda_{n}$ such that $w_{n}$ converges. Because of Proposition 4.20, $v_{\lambda_{n}}$ converges. Moreover, for all $\lambda$ and $\mu$, hypothesis $(\mathcal{H})$ implies that, denoting $C^{\prime}=\sup _{\lambda \in] 0,1]}\left\|v_{\lambda}\right\|$

$$
\begin{aligned}
\left\|v_{\lambda}-v_{\mu}\right\| & =\left\|\Phi\left(\lambda, v_{\lambda}\right)-\Phi\left(\mu, v_{\mu}\right)\right\| \\
& \leq\left\|\Phi\left(\mu, v_{\lambda}\right)-\Phi\left(\mu, v_{\mu}\right)\right\|+\left\|\Phi\left(\lambda, v_{\lambda}\right)-\Phi\left(\mu, v_{\lambda}\right)\right\| \\
& \leq(1-\mu)\left\|v_{\lambda}-v_{\mu}\right\|+|\lambda-\mu|\left(C+C^{\prime}\right)
\end{aligned}
$$

and thus that

$$
\left\|v_{\lambda}-v_{\mu}\right\| \leq\left|1-\frac{\lambda}{\mu}\right|\left(C+C^{\prime}\right)
$$

Since $\lambda_{n} \rightarrow 0$ and $\frac{1}{\lambda_{n}}-\frac{1}{\lambda_{n+1}} \rightarrow 0,\left|1-\frac{\lambda_{n+1}}{\lambda_{n}}\right|$ also converges to 0 . Together with inequality (4.17) and the fact that $v_{\lambda_{n}}$ converges it implies the convergence of $v_{\lambda}$ as $\lambda$ goes to 0 .

Conversely, if $v_{\lambda}$ converges, then Proposition 4.20 implies that the sequence $w_{n}$ defined by equation (4.15) converges as soon as $\lambda_{n}$ and $\frac{1}{\lambda_{n}}-\frac{1}{\lambda_{n+1}}$ tend to 0 .

As in the Section 3 (Ex. 3.2), there is an interpretation in terms of games with uncertain duration:

Example 4.22. Consider the case of a game with Shapley operator $J$. Let $\left\{\lambda_{n}\right\}$ be a sequence in $\left.] 0,1\right]$ and $w_{n}$ defined by equation (4.15). Then $w_{n}$ is the value of the following game with uncertain duration: with probability $\lambda_{n}$ the game stops after stage 1 , and the payoff is the payoff during stage 1 . With probability $1-\lambda_{n}$ there is no payoff during stage 1 but a transition, and game goes to stage 2 . Then, conditionally to the game going to stage 2, with probability $\lambda_{n-1}$ the game stops after stage 2 , and the payoff is the payoff during stage 2; and with probability $1-\lambda_{n-1}$ there is no payoff during stage 2 but a transition, and game goes to stage 3 . 
If the game goes to stage $n$, with probability $\lambda_{1}$ the payoff is the payoff during stage $n$ and with probability $1-\lambda_{1}$ the payoff is 0 .

Proposition 4.20 then states that if $\left\{\lambda_{n}\right\}$ is of slow variation, the value of this game with uncertain duration is close to the value of the $\lambda_{n}$-discounted game.

As a final remark to this section, notice the way in which we proved Proposition 4.20, with a back and forth process to continuous dynamics; it should be interesting to search another proof using only discrete time methods.

\section{Concluding Remarks}

- In this paper we proved that the asymptotic behavior of $v_{n}$ and $v_{\lambda}$ can be derived from the asymptotic behavior of solutions of some evolutions equations, namely (2.15) and (2.17). It should thus be interesting to determine which additional conditions on the nonexpansive operator $J$ may imply convergence of the solutions of these equations, and so convergence of $v_{n}$ and $v_{\lambda}$.

- Notice that Corollary 4.19 hints that $v_{\lambda}$ and $v_{n}$ should have the same asymptotic behavior for a wide class of nonexpansive operators, since the study of $\lim v_{n}$ seems to be a limit case of the study of $\lim v_{\lambda}$. Of interest is also Corollary 4.7 which gives a sufficient condition for existence of both $\lim v_{n}$ and $\lim v_{\lambda}$ as well as their equality.

- In Examples 3.2 and 4.22 we saw that some results that arose naturally during this paper have a nice interpretation in the framework of games with uncertain duration. In particular we showed that for specific types of uncertain duration, the value of those games behave asymptotically either as $v_{n}$ or $v_{\lambda}$ as the expected time played tends to infinity. Following $[19,20]$ it thus should be interesting to study uncertain duration more generally, hoping that some conditions on the Shapley Operator will provide convergence of values for more than just finitely repeated and discounted games.

Acknowledgements. This article was written during the course of my Ph.D. thesis. I would like to thank my advisor Sylvain Sorin as well as Jérôme Bolte, Juan Peypouquet and an anonymous referee for very helpful comments and references.

\section{REFERENCES}

[1] H. Attouch and R. Cominetti, A dynamical approach to convex minimization coupling approximation with the steepest descent method. J. Differ. Equ. 128 (1996) 269-275.

[2] R.J. Aumann and M. Maschler with the collaboration of R.E. Stearns, Repeated Games with Incomplete Information. MIT Press (1995).

[3] V. Barbu, Nonlinear Semigroups and Differential Equations in Banach Spaces. Noordhoff International Publishing (1976).

[4] T. Bewley and E. Kohlberg, The asymptotic theory of stochastic games. Math. Oper. Res. 1 (1976) 197-208.

[5] T. Bewley and E. Kohlberg, The asymptotic solution of a recursion equation occurring in stochastic games. Math. Oper. Res. 1 (1976) 321-336.

[6] H. Brézis, Opérateurs Maximaux Monotones et Semi-Groupes de Contractions dans les Espaces de Hilbert, Mathematical Studies 5. North Holland (1973).

[7] M.G. Crandall and T.M. Liggett, Generation of semi-groups of nonlinear transformations on general Banach spaces. Amer. J. Math. 93 (1971) 265-298.

[8] H. Everett, Recursive Games, in Contributions to the Theory of Games 3, H.W. Kuhn and A.W. Tucker Eds., Princeton University Press (1957) 47-78.

[9] S. Gaubert and J. Gunawardena, The Perron-Frobenius Theorem for homogeneous, monotone functions. T. Am. Math. Soc. 356 (2004) 4931-4950.

[10] J. Gunawardena, From max-plus algebra to nonexpansive maps: a nonlinear theory for discrete event systems. Theor. Comput. Sci. 293 (2003) 141-167.

[11] J. Gunawardena and M. Keane, On the existence of cycle times for some nonexpansive maps. Technical Report HPL-BRIMS95-003 Ed., Hewlett-Packard Labs (1995).

[12] T. Kato, Nonlinear semi-groups and evolution equations. J. Math. Soc. Japan 19 (1967) 508-520. 
[13] Y. Kobayashi, Difference approximation of Cauchy problems for quasi-dissipative operators and generation of nonlinear semigroups. J. Math Soc. Japan 27 (1975) 640-665.

[14] E. Kohlberg, Repeated games with absorbing states. Ann. Stat. 2 (1974) 724-738.

[15] E. Kohlberg and A. Neyman, Asymptotic behavior of nonexpansive mappings in normed linear spaces. Israel J. Math. 38 (1981) 269-275.

[16] E. Lehrer and S. Sorin, A uniform Tauberian theorem in dynamic programming. Math. Oper. Res. 17 (1992) $303-307$.

[17] I. Miyadera and S. Oharu, Approximation of semi-groups of nonlinear operators. Tôhoku Math. J. 22 (1970) $24-47$.

[18] J.-J. Moreau, Propriétés des applications "prox". C. R. Acad. Sci. Paris 256 (1963) 1069-1071.

[19] A. Neyman, Stochastic games and nonexpansive maps, in Stochastic Games and Applications, A. Neyman and S. Sorin Eds., Kluwer Academic Publishers (2003) 397-415.

[20] A. Neyman and S. Sorin, Repeated games with public uncertain duration process. (Submitted).

[21] S. Reich, Asymptotic behavior of semigroups of nonlinear contractions in Banach spaces. J. Math. Anal. Appl. 53 (1976) $277-290$.

[22] J. Renault, The Value of Markov Chain Games with Lack of Information on One Side. Math. Oper. Res. 31 (2006) 490-512.

[23] R. Rockafellar, Convex Analysis. Princeton University Press (1970).

[24] D. Rosenberg and S. Sorin, An operator approach to zero-sum repeated games. Israel J. Math. 121 (2001) $221-246$.

[25] S. Sorin, A First Course on Zero-Sum Repeated Games. Springer (2002).

[26] S. Sorin, Asymptotic properties of monotonic nonexpansive mappings. Discrete Events Dynamical Systems 14 (2004) 109-122.

[27] W. Walter, Differential and Integral Inequalities. Springer-Verlag (1970). 